\title{
ON THE DISTRIBUTION OF CERTAIN HUA SUMS, II *
}

\section{S. J. PATTERSON ${ }^{\dagger}$}

1. Introduction. Let $f(x)$ be a polynomial with integral coefficients and, for $c \geq 1$, let

$$
S(f(x), c)=\sum_{j(\bmod c)} e(f(j) / c)
$$

where $e(x)=\exp (2 \pi i x)$. In [5] we proved that for $A \in \mathbb{Z}, A \neq 0$ there is a constant $k(A)$ so that, for $\varepsilon>0$,

$$
\sum_{c \leq X} S\left(A x^{3}, c\right)=k(A) X^{\frac{4}{3}}+\mathcal{O}\left(X^{\frac{5}{4}+\varepsilon}\right)
$$

but without being able to give a simple formula for $k(A)$. The purpose of this note is to complete this result by giving an explicit formula for $k(A)$.

Theorem 1. For $A \neq 0$ we have

$$
k(A)=\frac{(2 \pi)^{2 / 3} \Gamma\left(\frac{1}{3}\right)}{24 \cdot L\left(2,\left(\frac{-3}{\cdot}\right)\right)} \prod_{p^{k} \| A} T_{p}\left(p^{k}\right) \cdot A^{-\frac{1}{3}}
$$

where

$$
\begin{aligned}
& T_{p}\left(p^{k}\right)=p^{[k / 3]}+p^{[(k-1) / 3]}-p^{-1} \quad \text { if } \quad k \geq 1, p \equiv 1 \quad(\bmod 3) \\
& =\left(p^{[k / 3]}+p^{[(k-1) / 3]}\right) \frac{1+p^{-1}}{1+p^{-2}}-\frac{2 p^{-1}}{1+p^{-2}} \quad \text { if } \quad k \geq 1, p \equiv 2 \quad(\bmod 3) \\
& =3^{\left[\frac{k}{3}\right]}+3^{\left[\frac{k-1}{3}\right]}-\frac{1}{3} \quad \text { if } \quad k \geq 1, p=3 \text {. }
\end{aligned}
$$

Note that

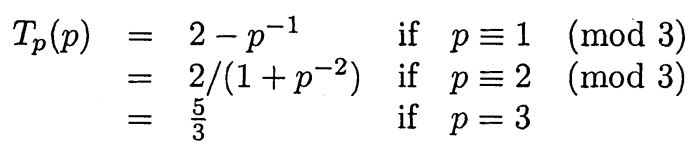

Numerically we have

$$
\frac{(2 \pi)^{2 / 3} \Gamma\left(\frac{1}{3}\right)}{24 L\left(2,\left(\frac{-3}{.}\right)\right)}=0.48646 \ldots .
$$

It should be noted that in [2, Theorem 1.3] R. Livné and the author proved an analogous theorem to the one above but now over $\mathbb{Z}[\omega]$ where $\omega$ is a third root of unity. One notices that the coefficients of leading term of this theorem and of the theorem above have the same general structure. One major difference, however, is

*Received December 14, 2001; accepted for publication October 25, 2002.

${ }^{\dagger}$ Mathematisches Institut, Bunsenstr. 3-5, 37073 Göttingen, Germany (sjp@uni-math.gwdg.de). 
that there is an extra logarithmic term in leading term of the asymptotic expansion of [2, Theorem 1.3]; the situation is analogous to the parallel theories of the divisor function $d(n)$ and $r(n)$, the number of representations of a number as a sum of two squares.

The proof of the theorem above is based on the methods of [5] but with variations in the details. It will be given in Section 2. In Section 3 we shall discuss some numerical experiments associated with the theorem.

Unfortunately, for some unaccountable reason, the author gave the formula in [5, Lemma 2.4] incorrectly; it should read, when $p \not X A$,

$$
S\left(A x^{3}, p\right)=g(A, \varepsilon, \pi)+\overline{g(A, \varepsilon, \pi)} .
$$

The factor of 2 is then found in many of the other formulae of the paper. In fact, although the formulae are thereby distorted, the general conclusion of the main theorem of [5] remains valid. The formula for the constant $k(A)$ given in [5] not only has to corrected; in the form given there it was not useful. It is the main purpose of this paper both to correct it and to bring it into a useful form. Additionally we give a brief report about some numerical experiments around the asymptotic formula.

2. Proof of the Theorem. We begin by considering the relevant analytic problem, namely the analytic behaviour of certain series, in a more general setting. These series, defined first in $\operatorname{Re}(s)>\frac{3}{2}$, are

$$
F(A, \chi, s)=\sum_{\substack{c=1 \\(c, f)=1}}^{\infty} S\left(A x^{3}, c\right) \chi(c) c^{-s}
$$

where $\chi$ is a primitive Dirichlet character of conductor $f$. We write $A$ as $3^{\alpha} \cdot A_{1} \cdot A_{2}$ where $A_{1}$ is made up of primes $\equiv 1(\bmod 3)$ and $A_{2}$ of primes $\equiv 2(\bmod 3)$. We write the series as

$$
\sum_{k=0}^{\infty} \sum_{c_{1}} \sum_{c_{2}} \sum_{c_{3}} S\left(3^{\alpha} A_{1} A_{2} \cdot x^{3}, 3^{k} c_{1} c_{2} c_{3}\right) \chi\left(3^{k} c_{1} c_{2} c_{3}\right) 3^{-k s} c_{1}^{-s} c_{2}^{-s} c_{3}^{-s}
$$

where $c_{1}$ is made up of primes $\equiv 1(\bmod 3), c_{2}$ of primes dividing $A_{2}$ and $c_{3}$ of primes $\equiv 2(\bmod 3)$ which do not divide $A_{2}$. We can, by [5, Lemma 2.1$]$, write this as:

$$
\begin{aligned}
\sum \sum \sum \sum & S\left(3^{\alpha} A_{1} A_{2} c_{1}^{2} c_{2}^{2} c_{3}^{2}, 3^{k}\right) 3^{-k s} \chi\left(3^{k}\right) \\
& S\left(3^{\alpha+2 k} A_{1} A_{2} c_{2}^{2} c_{3}^{2}, c_{1}\right) \cdot c_{1}^{-s} \chi\left(c_{1}\right) \\
& S\left(3^{\alpha+2 k} A_{1} A_{2} c_{1}^{2} c_{3}^{2}, c_{2}\right) c_{2}^{-s} \chi\left(c_{2}\right) \\
& S\left(3^{\alpha+2 k} A_{1} A_{2} c_{1}^{2} c_{2}^{2}, c_{3}\right) \cdot c_{3}^{-s} \chi\left(c_{3}\right) .
\end{aligned}
$$

In this sum it turns out that the internal sums over $c_{2}$ and $c_{3}$ are comparatively elementary. The sum over $c_{1}$ will lead to functions known from the theory of metaplectic forms. We begin by investigating the sums over $c_{2}$ and $c_{3}$. The last two lines can be replaced by 


$$
S\left(A_{2} x^{3}, c_{2}\right) \cdot c_{2}^{-s} \chi\left(c_{2}\right)
$$

and

$$
S\left(x^{3}, c_{3}\right) \cdot c_{3}^{-s} \chi\left(c_{3}\right)
$$

respectively since any $b$ coprime to $c_{2}$ resp. $c_{3}$ is of the form $\beta^{3}\left(\bmod c_{2}\right)$ resp. $\beta^{3}$ $\left(\bmod c_{3}\right)$. By $\left[5\right.$, Lemma2.3] we have for $c_{3}$ made up of primes $\equiv 2(\bmod 3)$,

$$
S\left(x^{3}, c_{3}\right)=\sum_{d_{1}^{2} d_{2}^{3} d_{3}^{4}=c_{3}} d_{1} d_{2}^{2} d_{3}^{2} \mu\left(d_{3}\right) .
$$

We can also treat the term in $c_{2}$ in a similar but more involved fashion. Let, for any integer $m, \operatorname{Supp}(m)$ be the set of primes dividing $m$. Then we consider, for any $c_{2}$ the subset

$$
\left\{p: p \mid A_{2}, \operatorname{ord}_{p}\left(c_{2}\right) \geq \operatorname{ord}_{p}\left(A_{2}\right)\right\}
$$

We combine all the terms of the sum where this set is a fixed subset $M$ of $\operatorname{Supp}\left(A_{2}\right)$, and have then a sum over $M$. For $M \subset \operatorname{Supp}\left(A_{2}\right)$ let

$$
\begin{aligned}
A_{2}(M) & =\prod_{p \in M} p^{\operatorname{ord}_{p}\left(A_{2}\right)}, \\
A_{2}^{*}(M) & =\prod_{p \mid A_{2}, p \notin M} p^{\operatorname{ord}_{p}\left(A_{2}\right)}, \\
c_{2}^{*}(M) & =\operatorname{gcd}\left(c_{2}, A_{2}^{*}(M)\right) \\
c_{2}(M) & =c_{2} / c_{2}^{*}(M) .
\end{aligned}
$$

If $\left\{p: p \mid A_{2}, \operatorname{ord}_{p}\left(c_{2}\right) \geq \operatorname{ord}_{p}\left(A_{2}\right)\right\}=M$ then

$$
\begin{aligned}
S\left(A_{2} x^{3}, c_{2}\right) & =c_{2}^{*}(M) \cdot A_{2}(M) \cdot S\left(x^{3}, c_{2}(M) / A_{2}(M)\right) \\
& =c_{2}^{*}(M) A_{2}(M) \sum_{d_{1}^{2} d_{2}^{3} d_{3}^{4}=c_{2}(M) / A_{2}(M)} d_{1}^{2} \cdot d_{2}^{2} \cdot d_{3}^{2} \mu\left(d_{3}\right)
\end{aligned}
$$

Note that for each $M \subset \operatorname{Supp}\left(A_{2}\right)$ the set of possible $c_{2}^{*}(M)$ is finite. The sum over $c_{2}$ then becomes an outer sum over $M$ and a inner sums over $c_{2}(M)$ and $c_{2}^{*}(M) \mid A_{2}^{*}(M)$.

We next consider the term in $c_{1}$. This we shall treat as above, but the details are more involved. Let $T \subset \operatorname{Supp}\left(A_{1}\right)$ and we shall consider such $c_{1}=c_{11} \cdot c_{12} \cdot c_{13}$ for which

$$
\begin{aligned}
& \operatorname{Supp}\left(c_{13}\right) \cap \operatorname{Supp}\left(A_{1}\right)=\emptyset \\
& \operatorname{ord}_{p}\left(c_{11}\right)<\operatorname{ord}_{p}\left(A_{1}\right), \quad p \in \operatorname{Supp}(a)-T \\
& \operatorname{ord}_{p}\left(c_{12}\right) \geq \operatorname{ord}_{p}\left(A_{1}\right) \quad p \in T \text {. }
\end{aligned}
$$

Then 


$$
S\left(3^{\alpha+2 k} A_{1} A_{2} c_{2}^{2} c_{3}^{2}, c_{1}\right)=A_{1}(T) \cdot c_{11} \cdot S\left(3^{\alpha+2 k} A_{1}^{*}(T) A_{2} c_{11}^{2} c_{2}^{2} c_{3}^{2},\left(c_{12} / A_{1}(T)\right) c_{13}\right)
$$

where $A_{1}(T)=\prod_{p \in T} p^{\operatorname{ord}_{p}\left(A_{1}\right)}$ and $A_{1}^{*}(T)=\prod_{p \mid A_{1} p \notin T} p^{\operatorname{ord}_{p}\left(A_{1}\right)}$. Also, if is coprime to 3 and $B$ is coprime to $c$ then by [5, Lemma 2.7]

$$
S\left(B x^{3}, c\right)=\sum_{\substack{\delta, d \\ N(\delta) \cdot d^{3}=c}} g(B, \varepsilon, \delta) \cdot d^{2}
$$

where $\delta \in \mathbb{Z}[\omega], \delta \equiv 1(\bmod 3)$ and $d \in \mathbb{N}$.

Finally we observe that for $B \not \equiv 0(\bmod 3)$

$$
\begin{aligned}
S\left(3^{\alpha} \cdot B, 3^{k}\right) & =3^{k} & & \text { if } \alpha \geq k \\
& =3^{\alpha} S\left(B, 3^{k-\alpha}\right) & & \text { if } \alpha<k .
\end{aligned}
$$

Next let $\chi_{9}$ be the primitive character of order 3 and conductor 9 given by

$$
\chi_{9}( \pm(1+3 v))=e\left(\frac{v}{3}\right)
$$

Then

$$
2 \cos \left(\frac{2 \pi B}{9}\right)=e\left(\frac{1}{9}\right) \cdot \chi_{9}(B)+e\left(-\frac{1}{9}\right) \overline{\chi_{9}}(B) .
$$

From [5, Lemma 2.3] we have for $B \not \equiv 0(\bmod 3)$

$$
\begin{aligned}
& S\left(B, 3^{l}\right)=3^{2 l / 3} \quad \text { if } l \equiv 0 \quad(\bmod 3) \\
& =0 \quad \text { if } l \equiv 1 \quad(\bmod 3) \\
& =3^{\frac{2 l-1}{3}}\left(1+e\left(\frac{1}{9}\right) \chi_{9}(B)+e\left(-\frac{1}{9}\right) \overline{\chi_{9}}(B)\right) \quad \text { if } l \equiv 2 \quad(\bmod 3) \text {. }
\end{aligned}
$$

Now we can combine all of these results. What we obtain is a sum over $M \subset$ $\operatorname{Supp}\left(A_{2}\right)$ and $T \subset \operatorname{Supp}\left(A_{1}\right)$ of

$$
\begin{aligned}
& \sum_{c_{11}, c_{2}^{*}(M)} \sum_{\delta, d} \sum_{d_{1}, d_{2}, d_{3}}\left(c_{11} \cdot c_{2}^{*}(M)\right)^{1-s} \chi\left(c_{11} \cdot c_{2}^{*}(M)\right)\left(A_{1}(T) A_{2}(M)\right)^{1-s} \chi\left(A_{1}(T) A_{2}(M)\right) \\
& g\left(c_{11}^{2} c_{2}^{*}(M) A_{1}^{*}(T) A_{2}^{*}(M), \varepsilon, \delta\right) N(\delta)^{-s} \chi(N(\delta)) d^{2-3 s} \chi\left(\delta^{3}\right) d_{1}^{1-2 s} d_{2}^{2-3 s} d_{3}^{2-4 s} \\
& \mu\left(d_{3}\right) \chi\left(d_{1}^{2} d_{2}^{3} d_{3}^{4}\right) \varepsilon\left(\left(\frac{d_{1}^{2} d_{3}}{\delta}\right)_{3}\right)\left\{\sum_{k=0}^{\alpha-1} 3^{k(1-s)} \chi\left(3^{k}\right) \varepsilon\left(\left(\frac{3^{k-\alpha}}{\delta}\right)_{3}\right)+\right. \\
& +\sum_{L=0}^{\infty} 3^{\alpha(1-s)} 3^{L(2-3 s)} \chi\left(3^{\alpha+3 L}\right)+\sum_{L=0}^{\infty} 3^{\alpha(1-s)-2 s+1} 3^{L(2-3 s)} \chi\left(3^{\alpha+2+3 L}\right) \cdot \varepsilon\left(\left(\frac{3^{2}}{\delta}\right)_{3}\right) \\
& \times\left(1+e\left(\frac{1}{9}\right) \chi_{9}\left(A_{1}^{*}(T) A_{2}^{*}(M) c_{11}^{2} c_{2}^{*}(M)^{2} N(\delta)^{2} d d_{1} d_{3}^{2}\right)+\right. \\
& \left.\left.+e\left(-\frac{1}{9}\right) \overline{\chi_{9}}\left(A_{1}^{*}(T) A_{2}^{*}(M) c_{11}^{2} c_{2}^{*}(M)^{2} \cdot N(\delta)^{2} d d_{1} d_{3}^{2}\right)\right)\right\}
\end{aligned}
$$


where $\operatorname{Supp}\left(d_{1}\right), \operatorname{Supp}\left(d_{2}\right)$ and $\operatorname{Supp}\left(d_{3}\right)$ are subsets of $\tilde{M}=\{p: p$ prime,$p \equiv$ $\left.2(\bmod 3), p \nmid A_{2}^{*}(M)\right\}$ and $\operatorname{Supp}(d)$ and $\operatorname{Supp}(N(\delta))$ are subsets of $\tilde{T}=\{p$ : $p$ prime $\left., p \equiv 1(\bmod 3), p \nmid A_{1}^{*}(T)\right\}$. The sums over $c_{11}$ and $c_{2}^{*}(M)$ are finite and are restricted as above. Now we observe that

$$
\sum_{d_{1} d_{3}^{2}=D} \mu\left(d_{3}\right)=|\mu(D)|
$$

we can therefore express the sum over $d_{2}$ as an Euler product. For convenience we write $c^{*}=c_{11} \cdot c_{2}^{*}(M), A(T, M)=A_{1}(T) A_{2}(M), A^{*}(T, M)=A_{1}^{*}(T) \cdot A_{2}^{*}(M)$. We then see that the expression above is the sum of five terms (with $D: \operatorname{Supp}(D) \subset \tilde{M}$ )

$$
\begin{aligned}
& \sum_{c^{*}, \delta, D}\left(c^{*} A(T, M)\right)^{1-s} \chi\left(c^{*} A(T, M)\right) \cdot|\mu(D)| \cdot D^{1-2 s} \chi(D)^{2} \\
& \sum_{k=0}^{\alpha-1} g\left(3^{\alpha-k} D c^{*^{2}} A^{*}(T, M), \varepsilon, \delta\right) N(\delta)^{-s} \cdot \chi(N(\delta)) \\
& \prod_{p \in \tilde{T} \cup \tilde{M}}\left(1-p^{2-3 s} \chi(p)^{3}\right)^{-1} \cdot 3^{k(1-s)} \chi\left(3^{k}\right) \\
& \sum_{c^{*}, \delta, D}\left(3^{\alpha} c^{*} \cdot A(T, M)\right)^{1-s} \chi\left(3^{\alpha} \cdot c^{*} \cdot A(T, M)|\mu(D)| D^{1-2 s} \chi(D)^{2}\right. \\
& g\left(D \cdot{c^{*}}^{2} \cdot A^{*}(T, M), \varepsilon, \delta\right) \cdot N(\delta)^{-s} \chi(N(\delta)) \\
& \prod_{p \in \tilde{T} \cup \tilde{M}}\left(1-p^{2-3 s} \chi(p)^{3}\right)^{-1} \cdot\left(1-3^{2-3 s} \chi(3)^{3}\right)^{-1} \\
& \sum_{c^{*}, \delta, D}\left(3^{\alpha} c^{*} A(T, M)\right)^{1-s} \chi\left(3^{\alpha} c^{*} A(T, M)\right) 3^{1-2 s} \chi(3)^{2} \\
& |\mu(D)| \cdot|D|^{1-2 s} \chi(D)^{2} \text {. } \\
& g\left(c^{*^{2}} 3 D A^{*}(T, M), \varepsilon, \delta\right) N(\delta)^{-s} \chi(N(\delta)) \\
& \prod_{p \in \tilde{T} \cup \bar{M}}\left(1-p^{2-3 s} \chi(p)^{3}\right)^{-1}\left(1-3^{2-3 s} \chi(3)^{3}\right)^{-1}
\end{aligned}
$$

and the two terms

$$
\begin{array}{ll}
e\left( \pm \frac{1}{9}\right) \quad & \sum_{c^{*}, \delta, D}\left(3^{\alpha} c^{*} A(T, M)\right)^{1-s} \chi\left(3^{\alpha} \cdot c^{*} \cdot A(T, M)\right) 3^{1-2 s} \chi(3)^{2} \\
& \chi_{9}\left(A^{*}(T, M) c^{*^{2}}\right)^{ \pm 1} \cdot|\mu(D)||D|^{1-2 s} \chi(D)^{2} . \\
& g\left(c^{*^{2}} 3 \omega^{ \pm 1} D A^{*}(T, M), \varepsilon, \delta\right) N(\delta)^{-s} \chi(N(\delta)) \\
& \prod_{p \in \tilde{T} \cup \tilde{M}}\left(1-p^{2-3 s} \chi(p)^{3}\right)^{-1} \cdot\left(1-3^{2-3 s} \chi(3)^{3}\right)^{-1}
\end{array}
$$

where we have used: $\chi_{9}(N(\delta))=\varepsilon\left(\left(\frac{\omega}{\delta}\right)_{3}\right)$.

We shall next show that the sum over $\delta$ can be continued to $\operatorname{Re}(s)>\frac{7}{6}$; we shall also determine the residue at $s=\frac{4}{3}$. What will also be evident from this discussion is that the series above are holomorphic in $\operatorname{Re}(s)>\frac{7}{6}$ if neither $\chi$ nor $\chi \cdot\left(\frac{-3}{.}\right)$ is of 
order 3. Since our main interest is to determine the residue at $s=\frac{4}{3}$ we shall not pursue this further.

In order to study the series

$$
\sum_{\delta} g(r, \varepsilon, \delta) N(\delta)^{-s} \chi(N(\delta))
$$

we define, for any set $R$ of primes of $\mathbb{Z}[\omega]$ excluding $\sqrt{-3}$ the function

$$
\psi_{R}(r, \chi, s)=\sum g(r, \varepsilon, \delta) N(\delta)^{-s} \chi(N(\delta))
$$

where $\delta \equiv 1(\bmod 3)$ and $\delta$ is not divisible by the primes in $R$. Then we have, if $Q$ is a further such set, $Q \cap R=\emptyset$ then if $r$ is of the form $r_{0} \delta_{1} \delta_{2}^{2}$ where $r_{0}$ is not divisible by any prime in $Q$, but $\delta_{1}$ and $\delta_{2}$ are square-free and divisible only by primes in $Q$. Then

$$
\begin{aligned}
\psi_{R \cup Q}(r, \chi, s)= & \prod_{\pi \in Q}\left(1-\chi(N(\pi))^{3} N(\pi)^{2-3 s}\right)^{-1} \\
& \sum_{d_{0}, d_{1}} \psi_{R}\left(r_{0} d_{0}\left(\delta_{1} / d_{1}\right) d_{2}^{2}, \chi, s\right) \chi\left(N\left(d_{0} d_{1}^{2}\right)\right) \\
& \frac{N\left(d_{1}\right) N\left(d_{0} d_{1}^{2}\right)^{-3} \mu\left(d_{0} d_{1}\right) g\left(r_{0}, \varepsilon, d_{0}\right)}{g\left(r_{0}, \varepsilon, d_{1}\right) \bar{\varepsilon}}\left(\left(\frac{\delta_{1} / d_{1}}{d_{0} d_{1}^{2}}\right)_{3}\right)
\end{aligned}
$$

see $[4, \S 2]$ for a rather more general formula. Here $d_{0}, d_{1} \equiv 1(\bmod 3), \operatorname{Supp}\left(d_{0}\right) \subset$ $Q-\operatorname{Supp}\left(\delta_{1} \delta_{2}\right)$ and $d_{1} / \delta_{1}$. Also we have

$$
\underset{s=\frac{4}{3}}{\operatorname{Res}} \psi_{R \cup Q}(r, \chi, s)=0 \quad \text { if }(\chi \circ N)^{3} \text { is non-principal }
$$

and

$$
\operatorname{Res}_{s=\frac{4}{3}} \psi_{R \cup Q}(r, 1, s)=\prod_{\pi \in Q}\left(1+N(\pi)^{-1}\right)^{-1} \cdot \operatorname{Res}_{s=\frac{4}{3}} \psi_{R}(r, 1, s)
$$

- see [1, p. 134] for a variant.

Next we recall, that if we use the estimates

and

$$
|g(r, \varepsilon, c)|=N(c)^{\frac{1}{2}} \quad \text { if } c \text { and } r \text { are coprime }
$$

$$
|g(r, \varepsilon, c)| \leq \varphi(c) \quad \text { in general }
$$

then, with the aid of the functional equation ([3, Theorem 6.1]) that, for any $\varepsilon>0$ there is $\varepsilon^{\prime}>0$ so that for $s: \frac{1}{2}-\varepsilon^{\prime}<\operatorname{Re}(s)<\frac{3}{2}+\varepsilon^{\prime},|\operatorname{Im}(s)| \geq 1$ one has

$$
\psi_{\{\lambda\}}(r, 1, s)=\mathcal{O}\left(N(r)^{\frac{3}{4}-\frac{\operatorname{Re}(s)}{2}+\varepsilon}|\operatorname{Im}(s)|^{3-2 \cdot \operatorname{Re}(s)+\varepsilon}\right)
$$


It follows that if $R$ is contained in the set of primes which cover split primes then in $\operatorname{Re}(s)>\frac{7}{6}$ we can let $Q$ exhaust the complete set of primes which divide the inert primes. Let $Q_{\infty}$ be the set of all such primes, i.e. primes in $\mathbb{Z}$ which are also primes in $\mathbb{Z}[\omega]$. Then we have additionally

$$
\underset{s=\frac{4}{3}}{\operatorname{Res}} \psi_{R \cup Q_{\infty}}(r, 1, s)=\prod_{q \equiv 2}\left(1+q^{-s}\right)^{-1} \underset{s=\frac{4}{3}}{\operatorname{Rod} 3)} \psi_{R}(r, 1, s) .
$$

We have (see, for example, $[5, \S 3]$ )

$$
\operatorname{Res}_{s=\frac{4}{3}} \psi_{R}(r, 1, s)=K \cdot \tau(r) / N(r)^{\frac{1}{6}} \prod_{\pi \in R}\left(1+N(\pi)^{-1}\right)^{-1}
$$

where

$$
K=\frac{(2 \pi)^{2 / 3} \Gamma\left(\frac{1}{3}\right)}{48 \sqrt{3} \zeta_{\mathbb{Q}(\omega)}(2)}
$$

Since we shall have to deal with the case where $r \in \mathbb{N}\left\{1, \omega, \omega^{2}\right\}$ we observe that $\tau(r) / N(r)^{\frac{1}{6}}$ is invariant modulo cubes; suppose $r_{1}$ is now coprime to 3 and is cube-free.

Then if $r_{1}$ is square-free

$$
\begin{array}{ll}
\tau\left(r_{1}\right) / N\left(r_{1}\right)^{\frac{1}{6}} & =2 \sqrt{3} r_{1}^{-\frac{1}{3}} \\
\tau\left(3 r_{1}\right) / N\left(3 r_{1}\right)^{\frac{1}{6}} & =2 \sqrt{3} r_{1}^{-\frac{1}{3}} \\
\tau\left(3 \omega r_{1}\right) / N\left(3 \omega r_{1}\right)^{\frac{1}{6}} & =2 \sqrt{3} e\left(-\frac{1}{9}\right) \overline{\chi_{9}\left(r_{1}\right)} r_{1}^{-\frac{1}{3}} \\
\tau\left(3 \omega r_{1}\right) / N\left(3 \omega^{2} r_{1}\right)^{\frac{1}{6}} & =2 \sqrt{3} e\left(+\frac{1}{9}\right) \chi_{9}\left(r_{1}\right) r_{1}^{-\frac{1}{3}}
\end{array}
$$

and for any other $r \in \mathbb{N} \cdot\left\{1, \omega, \omega^{2}\right\}$ not in a cube-class containing one of the above satisfies $\tau(r)=0$.

We shall now take set $R$ to be $\left\{\pi: \pi \mid A_{1}^{*}(T)\right\}$. We conclude that the (simple) pole of $F(A, s)=F(A, 1, s)$ at $s=\frac{4}{3}$ is a sum over $T, M$ of the five terms arising from the decomposition above. These are

$$
\begin{aligned}
& K \cdot \sum_{c^{*}, D}\left(c^{*} A(T, M)\right)^{-\frac{1}{3}}|\mu(D)| \\
& \times D^{-4 / 3} \sum_{k=0}^{\alpha-1} \tau\left(c^{*^{2}} 3^{\alpha-k} D A^{*}(T, M)\right) N\left(c^{*^{2}} 3^{\alpha-k} D A^{*}(T, M)\right)^{-\frac{1}{6}} \\
& \prod_{p \mid A_{1}^{*}(T)}\left(1+p^{-2}\right)^{-1} \cdot \prod_{(\bmod 3)}\left(1+q^{-2}\right)^{-1} \cdot 3^{-\frac{k}{3}} \cdot \prod_{p \in \tilde{T} \cup \tilde{M}}\left(1-p^{-2}\right)^{-1}, \\
& K \sum_{c^{*}, D}\left(3^{\alpha} c^{*} A(T, M)\right)^{-\frac{1}{3}}|\mu(D)| \cdot D^{-\frac{s}{3}} \tau\left(c^{*^{2}} \cdot D \cdot A^{*}(T, M)\right) N\left(c^{*^{2}} A^{*}(T, M)\right)^{-\frac{1}{6}} \\
& \prod_{p \mid A_{1}^{*}(T)}\left(1+p^{-s}\right)^{-1} \cdot \prod_{q \equiv 2}\left(1+q^{-2}\right)^{-1} \prod_{p \in \tilde{T} \cup \tilde{M}}\left(1-p^{-2}\right)^{-1},
\end{aligned}
$$




$$
\begin{aligned}
& K \sum_{c^{*}, D}\left(3^{\alpha} c^{*} A(T, M)\right)^{-\frac{1}{3}}|\mu(D)| D^{-5 / 3} \\
& \times 3^{-5 / 3} \tau\left(c^{*^{2}} 3 D A^{*}(T, M)\right) N\left(c^{*^{2}} 3 \cdot D \cdot A^{*}(T, M)\right)^{-\frac{1}{6}} \\
& \prod_{p \mid A_{1}^{*}(T)}\left(1+p^{-2}\right)^{-1} \cdot \prod_{q \equiv 2} \prod_{(\bmod 3)}\left(1+q^{-2}\right)^{-1} \cdot \prod_{p \in \tilde{T} \cup \tilde{M}}\left(1-p^{-2}\right)^{-1} \cdot\left(1-\frac{1}{9}\right)^{-1},
\end{aligned}
$$

and the two terms

$$
\begin{aligned}
& K \cdot e\left( \pm \frac{1}{9}\right) \sum_{c^{*}, D}\left(3^{\alpha} c^{*} A(T, M)\right)^{-\frac{1}{3}} \\
& \times|\mu(D)| D^{-5 / 3} \chi_{q}\left(A^{*}(T, M) c^{*^{2}}\right)^{2 \pm 1} \tau\left(c^{*^{2}} 3 \omega^{ \pm 1} D A^{*}(T, M)\right) \\
& N\left(c^{*^{2}} \cdot 3 \cdot \omega^{ \pm 1} \cdot D \cdot A^{*}(T, M)\right)^{-\frac{1}{6}} \prod_{p \mid A_{1}^{*}(T)}\left(1+p^{-2}\right)^{-1} \prod_{q \equiv 2} \prod_{(\bmod 3)}\left(1+q^{-2}\right)^{-1} \\
& \prod_{p \notin \tilde{T} \cup \tilde{M}}\left(1-p^{-2}\right)^{-1}\left(1-\frac{1}{9}\right)^{-1} .
\end{aligned}
$$

These expressions now simplify. First of all in view of the formulae for $\tau$ we see that the last three terms are all equal. Also we can carry out the sums over $D$. These now lead to three terms:

$$
\begin{aligned}
& K \sum_{c^{*}}\left(c^{*} A(T, M)\right)^{-\frac{1}{3}} \prod_{p \mid A^{*}(T, M)} \frac{1-p^{-2}}{1+p^{-2}} \cdot 3^{-\frac{k}{3}} \cdot \prod_{p_{1} \neq 3}\left(1-p_{1}^{-2}\right)^{-1} \\
& \sum_{k=0}^{\alpha-1} \tau\left(c^{*^{2}} A^{*}(T, M) \cdot 3^{\alpha-k}\right) N\left(c^{*^{2}} A^{*}(T, M) 3^{\alpha-k}\right)^{-\frac{1}{6}} \\
& K \sum_{c^{*}}\left(3^{\alpha} c^{*} A(T, M)\right)^{-\frac{1}{3}} \tau\left(c^{*^{2}} A^{*}(T, M)\right) N\left(c^{*^{2}} A^{*}(T, M)\right)^{-\frac{1}{6}} \\
& \prod_{p \mid A^{*}(T, M)} \frac{1-p^{-2}}{1+p^{-2}} \prod_{p_{1} \neq 3}\left(1+p_{1}^{-2}\right)^{-1} .
\end{aligned}
$$

and

$$
\begin{aligned}
& K \cdot \sum_{c^{*}}\left(3^{\alpha} c^{*} A(T, M)\right)^{-\frac{1}{3}} 3^{-\frac{2}{3}} \tau\left(3 \cdot c^{*^{2}} A^{*}(T, M)\right) N\left(3 c^{*} A^{*}(T, M)\right)^{-\frac{1}{6}} \\
& \prod_{p \mid A^{*}(T, M)} \frac{1-p^{-2}}{1+p^{-2}} \cdot \prod_{p_{1} \neq 3}\left(1-p_{1}^{-2}\right)^{-1} .
\end{aligned}
$$

In view of the definitions above and the evaluation of $\tau$ given above we see that $k(A) / k(1)$ is multiplicative. The evaluation of the primary factors is a laborious but entirely routine calculation which we shall not reproduce here.

The author does not understand why the method used in [5] appears less effective in finding a calculable expression for $k(A)$. It may merely be a matter of incompetence, but it seems that by making use of all the available relationships between the $\psi_{R}$ one reduces the evaluation of $\tau$ to a set where this function is much more manageable than in general.

3. Remarks. It is instructive to compare the results above with experimental determinations of $\sum_{c \leq X} S\left(A x^{3}, c\right) / X^{\frac{4}{3}}$ for large $X$. In practice "large" means of the 
order of $10^{5}$. The calculation of $S\left(A x^{3}, c\right)$ involves $\mathcal{O}(c \log c)$ steps and consequently the calculation of the sum above requires of the order $X^{2} \log X$ steps. As the amount of information gained, measured in the number of decimal digits which can be estimated with any certainty, presumably grows as $\log X$ (i.e. the error term is of the form $\mathcal{O}\left(X^{-\eta}\right)$ with some $\eta>0$. there is little to be gained by extending the calculation from, say $10^{5}$ to $2 \cdot 10^{5}$. Whereas the cost increases fourfold the increase in $\log X$ and, so essentially in the information gained is perhaps around $6 \%$. The formula of the Theorem gives

$$
k(1)=\frac{(2 \pi)^{2 / 3} \Gamma\left(\frac{1}{3}\right)}{24 \cdot L\left(2, \chi_{-3}\right)}=0.48646 \ldots
$$

and

$$
\begin{aligned}
& k(2)=\frac{8}{5} k(1) \cdot 2^{-\frac{1}{3}}=0.6177 \ldots \\
& k(3)=\frac{5}{3} k(1) \cdot 3^{-\frac{1}{3}}=0.5621 \ldots \\
& k(4)=\frac{8}{5} k(1) \cdot 4^{-\frac{1}{3}}=0.4903 \ldots \\
& k(5)=\frac{25}{13} k(1) \cdot 5^{-\frac{1}{3}}=0.5470 \ldots \\
& k(6)=\frac{8}{3} k(1) \cdot 6^{-\frac{1}{3}}=0.7138 \ldots \\
& k(7)=\frac{13}{7} k(1) \cdot 7^{-\frac{1}{3}}=0.4722 \ldots \\
& k(8)=\frac{14}{5} k(1) \cdot 8^{-\frac{1}{3}}=0.6810 \ldots \\
& k(9)=\frac{5}{3} k(1) \cdot 9^{-\frac{1}{3}}=0.3897 \ldots
\end{aligned}
$$

The corresponding experimental values $\left(X=10^{5}\right)$ are

$$
\begin{array}{rlr}
A & =1 & 0.4765 \\
& =2 & 0.6081 \\
& =3 & 0.5633 \\
& =4 & 0.4936 \\
& =5 & 0.5514 \\
& =6 & 0.7088 \\
& =7 & 0.4508 \\
& =8 & 0.6468 \\
& =9 & 0.4046
\end{array}
$$

The accuracy given in the second list is not meaningful as it stands as small variations in $X$ give rise to much larger variations than the data of the last two decimal places given. This can be seen from Fig.2. It is merely given for the sake of comparison with the first list. The corresponding convergence can be seen, in the case $A=1$ from the graph of $X^{-\frac{4}{3}} \sum_{c \leq X} S\left(x^{3}, c\right)$ for the range $x \leq 10^{5}$ (Fig.1), sampled (linearly) at multiples of 500 . In this case a closer look at the convergence is afforded by Fig.2. In several cases, $A=1,2,4,7$ and 8 in the range $X \leq 10^{5}$ the error term is of a fixed sign over a long interval, as in the case shown. In the cases $A=1,2,7$ and 8 it is negative, in the case $A=4$ it is positive. In view of [5, Theorem 2.8] and [3, Theorem 6.1] it seems plausible that $F(A, s)$ has poles at the zeros of $L\left(3 s-2,\left(\frac{-3}{.}\right)\right)$, and thus in the half-plane $\operatorname{Re}(s) \geq \frac{5}{6}$. We therefore expect that the error term in $\sum_{c \leq X} S\left(A x^{3}, c\right)-k(A) X^{\frac{4}{3}}$ cannot be $O\left(X^{\frac{5}{6}}\right)$; the same would hold for Riesz means. If, 


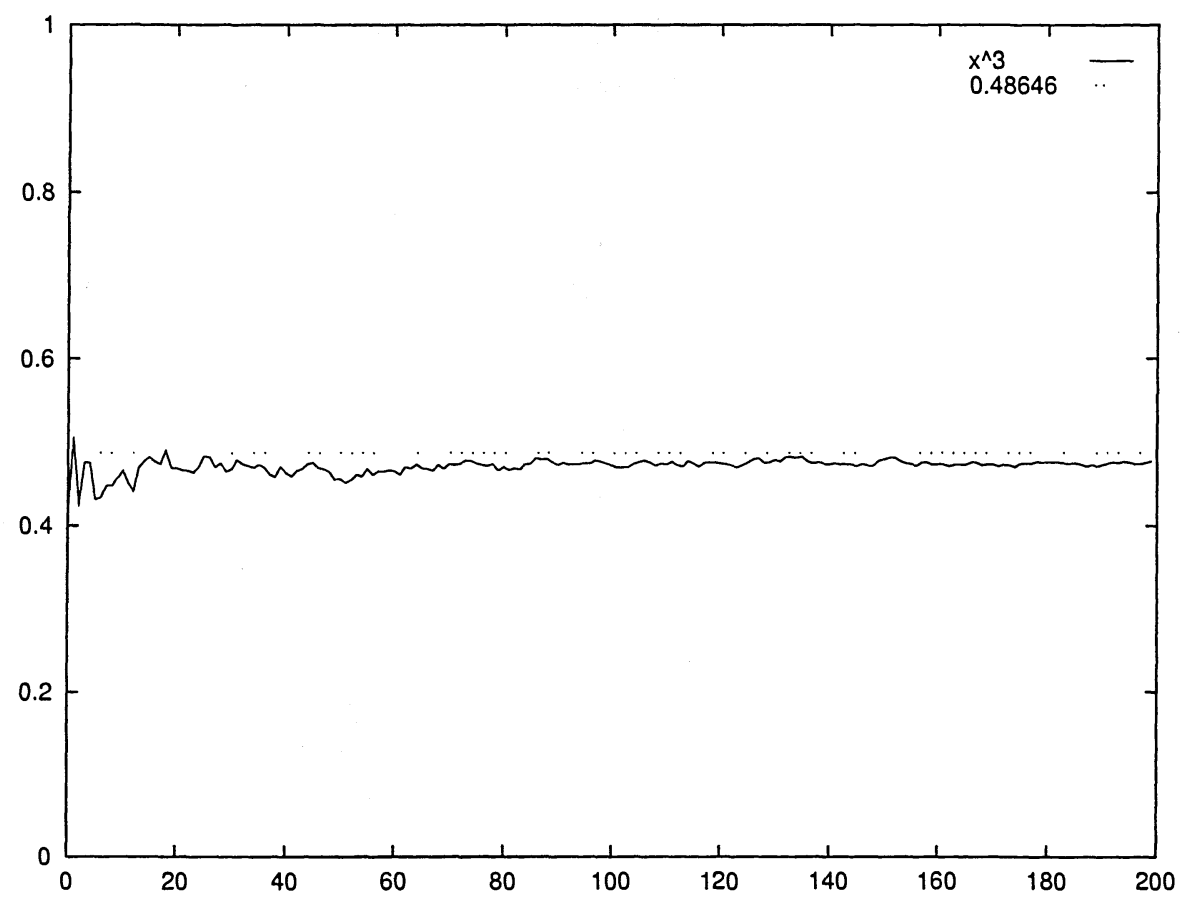

FIG. 1. The convergence in the case of $x^{3}$.

as one would expect, most of the zeros of $L\left(3 s-2,\left(\frac{-3}{-}\right)\right)$ yield poles of $F(A, s)$ then one would have that the spectrum of $X^{-\frac{5}{6}}\left(\sum_{c \leq X} S\left(A x^{3}, c\right)\right)$ considered as a function of $\xi$ with $X=\exp (\xi)$ would contain so many high-frequency components that it would presumably be impossible to improve the estimates by suppressing them by means of a "filter", nor does it appear feasible to estimate them numerically and to subtract them ("anti-noise"). From the point of of view of analytic number theory one would need a summation formula such as Riemann's explicit formula or one of Voronoi's type to do better and these do not exist at present. In other words, there seems to be no method, in the present state of knowledge, of obtaining a better estimate for $k(A)$ from the knowledge of the $S\left(A x^{3}, c\right)$ with $c \leq X$ than that given by $X^{-\frac{4}{3}} \sum_{c \leq X} S\left(A x^{3}, c\right)$. This is regrettable as one also expects that for an arbitrary integral cubic polynomial $f$ the asymptotic expansion

$$
\sum_{c \leq X} S(f(x), c) \sim k_{f} \cdot X^{\frac{4}{3}}
$$

where

$$
S(f(x), c)=\sum_{j} e\left(\frac{f(j)}{c}\right) .
$$

In view of the above, one cannot expect to be able to estimate $k_{f}$ well with a reasonable amount of calculation. The results above show that, even when $A_{1}$ and 


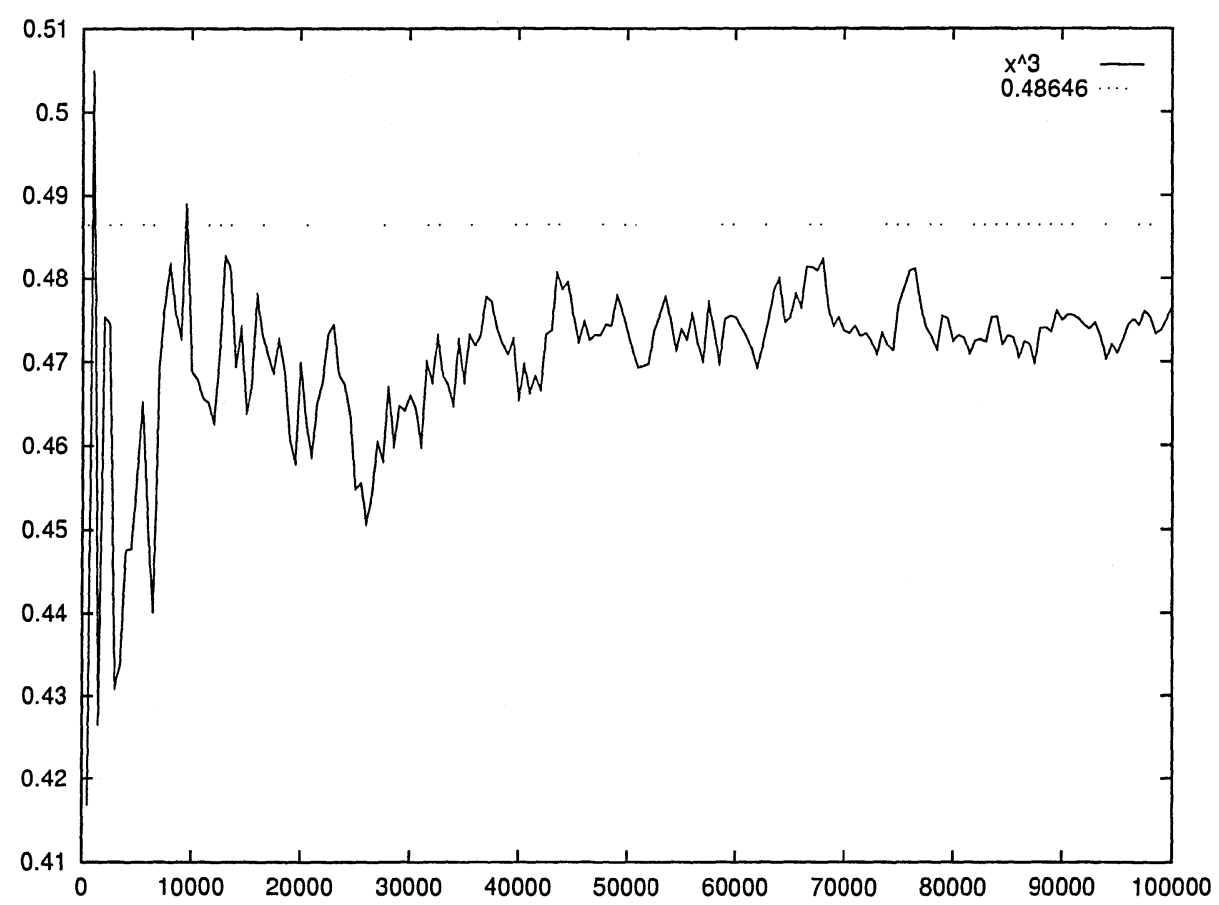

FIG. 2. The convergence in the case of $x^{3}$ examined more closely.

$A_{2}$ are such that $A_{1} / A_{2}$ is a rational cube, $k\left(A_{1}\right) / k\left(A_{2}\right)$ can be a relatively complex rational number which we could not recognize from experimental evidence.

Finally, there were a number of errors and misprints in [5] which we shall now correct. The most serious was in Lemma 2.4 which we noted above. Further corrections are:

In Lemma 2-3 the sign of $2 \cos \frac{2 \pi A}{9}$ should be "+", not "-".

In the first line of the proof of Lemma 2.6 the formula should be

$$
\sum t(A, c) c^{-s}
$$

with an analogous correction three lines later.

In $\S 3$, definition of $\tau$ : in the third case a "=" is missing.

\section{REFERENCES}

[1] D.A. Kazhdan, S.J. Patterson, Metaplectic Forms, Pub. Math. St/ES, 59 (1984), pp. 35-142

[2] R. LIVNÉ, S.J. PATTERSON, The first moment of cubic exponential sums, Inv. Math., 148 (2002), pp. 79-116.

[3] S.J. PAtterson, A cubic analogue of the theta series, J. reine angew. Math., 296 (1977), pp. 125-161, pp. 217-220.

[4] S.J. Patterson, The distribution of general Gauss sums and similar functions at prime arguments, Proc. Lond. Math. Soc., (3) 54 (1987), pp. 193-215.

[5] S.J. Patterson, On the distribution of certain Hua sums, Asian J. Math., 4 (2000), pp. 977986. 
